Received: 2021.03.11 Accepted: 2021.07.13 Available online: 2021.07 .31 Published: 2021.09.06

Authors' Contribution: Study Design A Data Collection B Statistical Analysis C Data Interpretation D Manuscript Preparation E Literature Search F Funds Collection G

Corresponding Author: Conflict of interest:

\title{
Renal Cell Carcinoma Mimicking Transitional Cell Carcinoma: A Case Report
}

BeF 1,2 Wiwan Irama

в 3 Jin Kiat Teo

B 4 Kang Min Wong
1 Department of Diagnostic Radiology, Changi General Hospital, Singapore, Singapore

2 Department of Radiology, Wan Fang Hospital, Taipei Medical University, Taipei, Taiwan

3 Department of Urology, Changi General Hospital, Singapore (Yong Loo Lin School of Medicine, NUS), Singapore, Singapore

4 Department of Diagnostic Radiology, Changi General Hospital, Singapore (DukeNUS Medical School), Singapore, Singapore

Wiwan Irama, e-mail: b94401121@gmail.com

None declared

\begin{abstract}
Patient: Female, 76-year-old
Final Diagnosis: Renal cell carcinoma

Symptoms: $\quad$ Flank pain - haematuria

Medication:

Clinical Procedure:

Nephroureterectomy

Specialty: Radiology • Urology

Objective: Unusual clinical course

Background: Preoperative differentiation between renal cell carcinoma (RCC) and transitional cell carcinoma (TCC) is of utmost important for determining surgical strategy, whether nephrectomy or nephro-ureterectomy, as well as the necessity for wider lymphadenectomy and subsequent intensive surveillance, as the latter is more prone to recurrence.

Case Report: A 76-year-old Chinese woman presented with flank pain and gross hematuria, and was found to have rightsided hydronephrosis. An obstructing tumor in the renal pelvis was shown on a computed tomography (CT) intravenous pyelogram. Although its enhancement pattern was suggestive of RCC, the location within the collecting system without any attachment to the renal parenchyma is very unusual. The mass was diagnosed histopathologically as RCC on both ureteroscopic biopsy and subsequent radical nephrectomy.

Conclusions: We present a rare case of RCC growing exclusively in the renal pelvis, mimicking a TCC. Hypotheses regarding this unusual presentation include direct invasion, continuous implantation, and intraluminal transit down the collecting system. The characteristics on imaging studies, including greater enhancement and higher tumorto-kidney attenuation ratio, may provide a clue for diagnosis, but ureteroscopy and histopathology are the criterion standards and should be considered as part of routine preoperative assessment. Amidst controversies and inconsistencies, more and more emerging evidence suggests that RCC with urinary collecting system invasion is associated with less favorable overall and recurrence-free survival, especially in localized diseases.
\end{abstract}

Keywords: Carcinoma, Transitional Cell $・$ Diagnosis, Differential $・$ Renal Cell Carcinoma 1

Full-text PDF: https://www.amjcaserep.com/abstract/index/idArt/932098

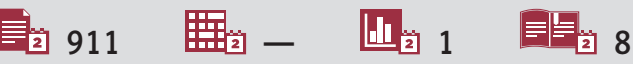




\section{Background}

Preoperative differentiation between renal cell carcinoma (RCC) and transitional cell carcinoma (TCC) is of utmost importance for determining type of surgery, nephrectomy or nephroureterectomy, and the necessity for more extensive lymphadenectomy. Intensive surveillance for metachronous tumors in the remnant urinary tract is often needed for patients with TCC.

The imaging features and enhancement pattern on computed tomography (CT) may provide a clue for diagnosis, but ureteroscopy and histopathology results should be considered as the criterion standards and routine preoperative assessment.

An RCC growing exclusively in the renal pelvis, as presented in the current case report, is very rare. Only a few similar cases have been published in the literature, the most recent one being fumarate hydratase-deficient RCC arising in a patient suffering from hereditary leiomyomatosis and renal cell carcinoma (HLRCC) syndrome.

\section{Case Report}

A 76-year-old Chinese woman with no underlying medical problem presented to the Emergency Department with 1-day duration of intermittent right flank pain and gross hematuria. She had no fever or dysuria, and a renal punch was negative. Bedside ultrasound showed moderate right hydronephrosis.

On CT intravenous pyelogram, a $1.6-\mathrm{cm}$ lobulated mass was seen in the right renal pelvis. It was mildly hyperdense (38 $\mathrm{HU}$ ) on pre-contrast images (Figure $1 \mathrm{~A}$ ), heterogeneously enhanced on nephrographic phase $(78 \mathrm{HU})$ but less than that of normal parenchyma $(121 \mathrm{HU})$ with attenuation ratio of 0.64 (Figure 1B-1D). No malignant cells were found on urine cytology. With a tentative diagnosis of collecting system tumor, she underwent ureteroscopy, where a renal pelvic mass was confirmed and biopsied. Histologically, the lesion harbored carcinoma cells in nests and tubules, polygonal to round with pleomorphic and hypochromatic nuclei. Expression of paired-box gene 8 (PAX8) was noted. Napsin A, 34betaE12, and cytokeratin 7 (CK7) were positive. Tumor protein p63 and GATA-binding protein 3 (GATA3, markers for TCC) were negative, while alpha-methylacyl-CoA racemase (AMACR) was focally positive.

On subsequent whole-body staging $\mathrm{CT}$, the mass showed interval growth with progressed hydronephrosis. There was no thoracoabdominal lymphadenopathy, or evidence of osseous metastasis on bone scan. The tumor remained confined to the kidney (pT1a) when radical nephrectomy was performed. It had a growth pattern resembling TCC, predominantly tubular, and tubulopapillary architecture and lack of demonstrable attachment to renal parenchyma. It was, however, proven to be of renal origin by positive PAX8/napsin A and absence of p63/GATA3.

\section{Discussion}

TCC accounts for $90 \%$ of all cancers in the renal pelvis, so the discovery of a renal mass with its epicenter in the pelvicaliceal system is generally considered TCC. In histopathologic studies, up to $14 \%$ of RCC demonstrate involvement of the collecting system [1]. RCC that exclusively grows within the collecting system, however, is exceedingly rare. A similar case has been recently reported Ajjikuttira et al in a patient with hereditary leiomyomatosis and renal cell carcinoma (HLRCC) syndrome [2].

There are 3 hypotheses of how RCC manifests as renal pelvic or juxtapelvic mass. First, invasion into hollow structure is easier compared to solid parenchyma when tumor arises from marginal area. Second, implantation of RCC cells through invasion of urothelial mucosa followed by intraluminal expansive growth. Third, tumor cells metastasizing via intraluminal transit down the urinary tract $[3,4]$. Considering the absence of dysplasia in the contiguous urothelial cells surrounding the $\mathrm{RCC}$ in our case, it might have taken the third route.

Dynamic CT is valuable for characterization of renal pelvic lesion. A high-density mass on pre-contrast scan may indicate calculus, blood clot, or neoplasm. Presence of enhancement (>15 HU) confirms a neoplastic etiology. Three-dimensional CT intravenous pyelogram delineates precise location of the mass and its association with renal parenchyma [3]. Various subtypes of RCC have minor differences, but it is generally agreed that RCC, being a hypervascular tumor, tends to enhance more than TCC [5]. Pal Bata et al observed significant difference between attenuation ratios of RCC and TCC compared to renal parenchyma in corticomedullary ( 0.77 vs 0.50$)$ and nephrographic (0.72 vs 0.45 ) phases [6], implying that RCC is likely to exhibit greater isodensity than a normal kidney. This phenomenon was observed in our case, where the tumor-to-kidney attenuation ratio was 0.64 on nephrographic phase. In cases where features of RCC such as necrosis, cystic degeneration, hemorrhage, and calcification are absent, such an enhancement pattern may suggest an alternative diagnosis.

Preoperative differentiation between RCC and TCC is crucial in determining the management strategy: nephrectomy or nephroureterectomy. Often, TCC necessitates wider lymphadenectomy and intensive surveillance of a metachronous tumor in the remaining urinary tract. Although there are some inconsistencies in the reports regarding oncological outcomes of patients with RCC invading the urinary collecting system, more and more evidence suggests that it has a negative impact on 


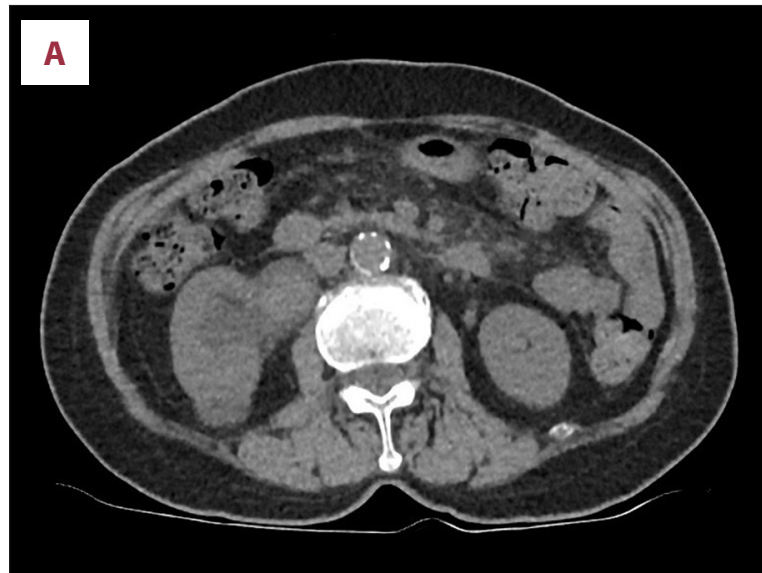

\section{B}

B
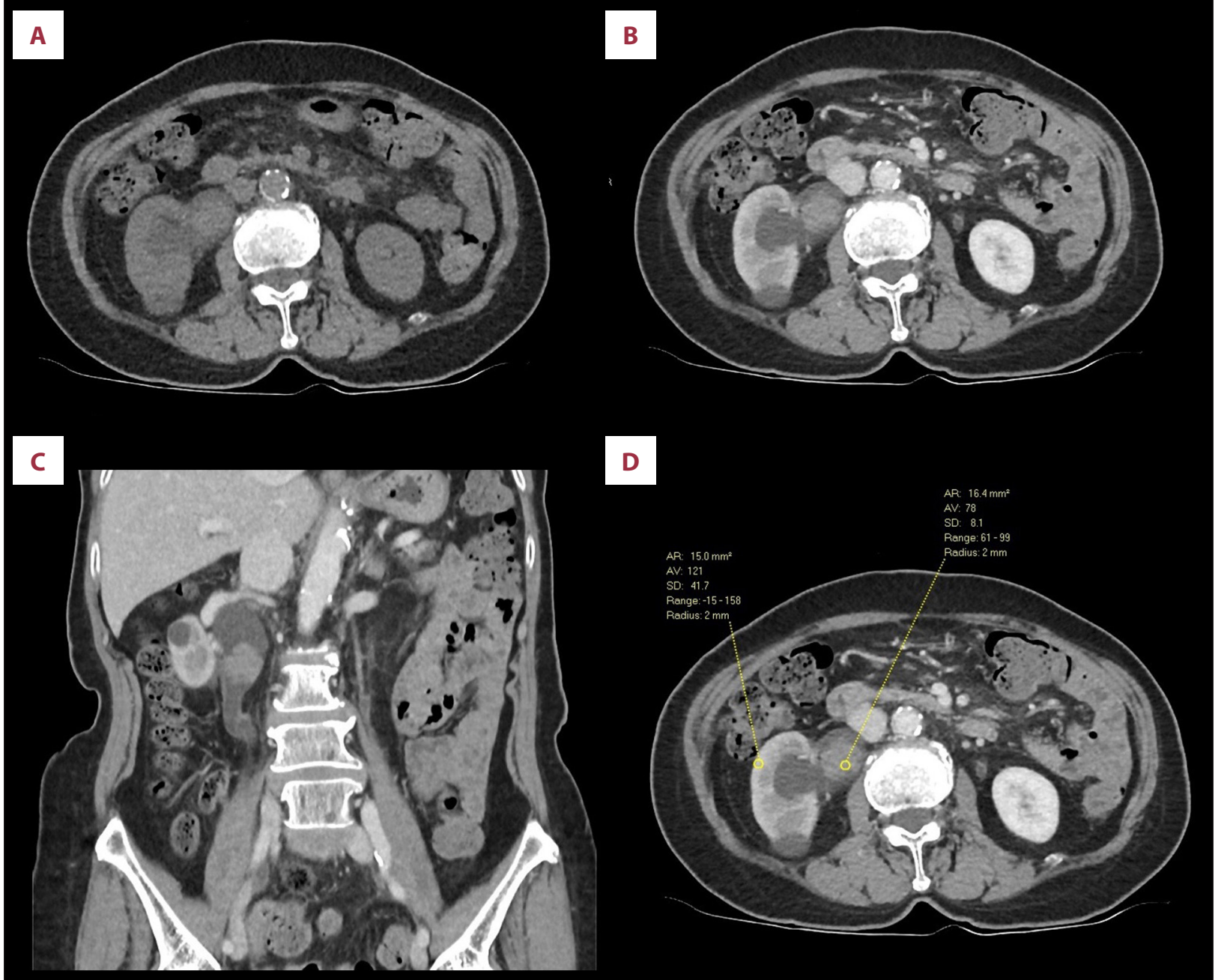

AR: 121
SD: 41.7
Range: $-15-1$
Raadius: 2 SD: 41.7
Range: $-15-158$
Redius: $2 \mathrm{~mm}$

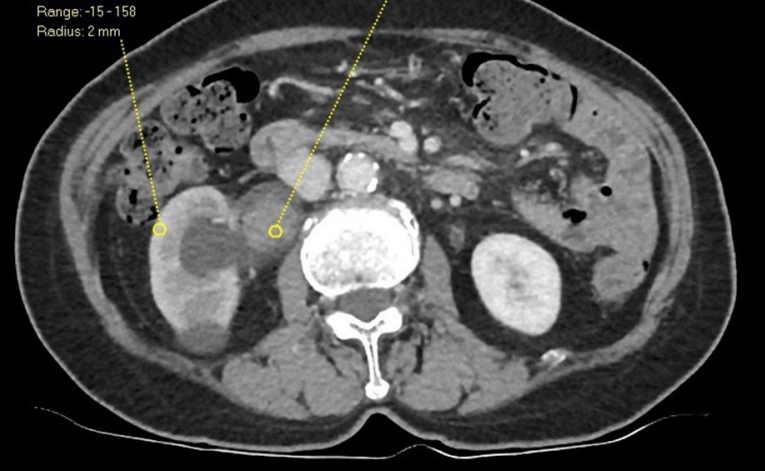

Figure 1. (A) Pre-contrast and (B, C) post-contrast axial and coronal CT intravenous pyelogram in nephrographic phase. A lobulated mass seen in the right renal pelvis, with post-contrast enhancement that is less than that of normal renal parenchyma. There is associated mild hydronephrosis. HU measurement is shown in (D), with a tumor-to-kidney attenuation ratio of 0.64.

overall survival and recurrence-free survival, especially in localized disease $[7,8]$. The importance of ureteroscopy and histopathology, in addition to various imaging studies, is therefore emphasized and should be considered a routine preoperative assessment.

\section{Conclusions}

Differentiating between RCC and TCC is important for surgical decision making. Often, RCC exhibits characteristic imaging features, with a higher tumor-to-kidney attenuation ratio. In rare cases, however, RCC grows exclusively in the collecting system and poses a difficulty to diagnosis. We therefore recommend ureteroscopy and histopathology to be part of routine preoperative assessment.

\section{Department and Institution Where Work Was Done}

Department of Diagnostic Radiology, Changi General Hospital, Singapore.

\section{Conflict of Intetests}

None declared.

\section{Declaration of Figures Authenticity}

All figures submitted have been created by the authors who confirm that the images are original with no duplication and have not been previously published in whole or in part. 


\section{References:}

1. Mary TK, Fergus VC, David MN, et al. CT of unusual renal masses invading the pelvicaliceal system: potential mimics of upper tract transitional cell carcinoma. Clin Imaging. 2011;35:77-80

2. Ajjikuttira A, Sharma P, Joshi A, et al. Fumarate hydratase-deficient renal cell carcinoma presenting as pelvicalyceal filling defect: An unusual presentation of a rare disease. BMJ Case Reports CP. 2021;14:e239331

3. Li YF, Ding Y, Chen DQ, et al. Renal cell carcinoma growing into the renal pelvis and mimicking transitional cell carcinoma: A case report and literature review. Onco Letters. 2015;9:1869-72

4. Shigenori K, Haruki K, Yoshikazu H, et al. Case report: Renal cell carcinoma with intraluminal spread of the entire upper urinary tract. Case Rep Med. 2013;2013:371387
5. Levine E. Computed tomography of renal masses. CRC Crit Rev Diagn Imaging. 1985;24(2):91-200

6. Pal B, David LT, Adam DT, et al. Transitional cell and clear cell renal carcinoma: Differentiation of distinct histological types with multiphasic CT. Acta Rad. 2014;55(9):1112-19

7. Chen L, Li H, Gu L, et al. Prognostic role of urinary collecting system invasion in renal cell carcinoma: A systematic review and meta-analysis. Sci Rep. 2016;6:21325

8. Bailey GC, Boorjian SA, Ziegelmann M, et al. Urinary collecting system invasion is associated with poor survival in patients with clear-cell renal cell carcinoma. BJU Int. 2017;119:585-90 